\title{
Moyamoya disease complicated by a bleeding distal anterior choroidal artery aneurysm: Approach, management and review of literature
}

\author{
Hekmat Zarzour* \\ Vickie and Jack Farber Institute for Neuroscience Thomas Jefferson University Hospital, USA
}

\begin{abstract}
Introduction: Moyamoya disease can be associated with a distal anterior choroidal artery (AChA) aneurysm. Subarachnoid hemorrhage resulting from distal AChA rupture in the setting of Moyamoya disease is a rare but increasingly challenging disease process.

Objective: To describe this disease entity and discuss the pathophysiology of aneurysmal occurrence, risk of re-bleeding, treatment options and safe zone of the AChA.

Methods: A middle-aged patient presented to the ED with complaints of worst headache of life, otherwise the patient was intact. Computed tomography (CT) scan of the head revealed intraventricular hemorrhage. A cerebral angiogram was performed and revealed a ruptured distal AChA aneurysm in the setting of Moyamoya disease. That increased in size on repeat angiography and required intervention.

Results: Cerebral angiography revealed a distal AChA aneurysm, conservative treatment was suggested to be followed by bypass. Repeat angiogram on day 3 after admission revealed increasing in size requiring endovascular embolization of the aneurysm using 0.3 cc of Onyx 34 . MRI revealed a small stroke in collaterals of AChA distribution in distal L MCA region. The patient did well.

Conclusion: Onyx embolization of distal AChA aneurysm associated with moyamoya disease is safe approach in situation of high risk of rebleeding; embolization of AChA distal to choroidal fissure seems to be safe acceptable approach, long occlusion of the anterior choroidal artery with catheter can cause permanent damage similar to long temporary clipping.
\end{abstract}

\section{Introduction}

Moyamoya disease, in the majority of cases, is a bilateral, progressive, spontaneous occlusion of the tip of internal carotid artery (ICA), or junction between the ICA, the proximal M1 segment of the middle cerebral artery (MCA), and the proximal A1 segment of the anterior cerebellar artery (ACA). The lateral and medial lenticulostriate branches and other perforaters supplying the ACA and MCA territories as the disease progress are small arteries that cannot endure the high demand required by the distal MCA and ACA territories in the context of an ischemic progression [1]. Increased flow in these perforators results in increased hemodynamic load and a risk of leading to pressure gradient aneurysm. The annual risk of aneurysmal bleeding is around $7 \%$, with a high associated morbidity and mortality[2]. The anterior choroidal artery (AChA) cisternal segment has multiple perforators as well that can be damaged during aggressive manipulation or predisposed towards spasm. Occluding the aneurysm with preserving the feeding artery is the goal; however, in many cases of AChA aneurysms this is not possible due to anatomical constraints. In such cases, bypass may be the best etiological treatment by preventing ischemic changes, reversing hyperdynamic flow, and facilitating regression of the aneurysm. If this fails, embolization or clipping can be best option despite the higher risk for ischemia and overall harm.

\section{Case report}

Middle-aged patient with no past medical history who was sitting in church and experienced the worse headache of his life, primarily occipital in location, prompting transfer to a nearby emergency department. Subsequent head CT (Figure 1) revealed intra-ventricular hemorrhage. Further testing with MRI/MRA (Figure 2) revealed features consistent with Moyamoya syndrome/disease. The patient was taken for angiogram, which confirmed Left MCA occlusion with left ICA ending in prominent lenticulostriate arteries consistent with Moyamoya features, and an aneurysm of the distal AChA (Figure 3). Throughout this the patient was neurologically intact and was admitted to the ICU for conservative treatment to be followed by bypass after recovery from hemorrhage. However, a repeat angiogram performed to determine any changes in aneurysm size on post-bleed day 3 revealed an increase the aneurysm size (from 3.5 to $5 \mathrm{~mm}$ ). (Figure 4).

The patient was intubated and a SL10 catheter was advanced into the AChA over a Synchro microwire. However, minimal resistance was encountered, and the SL 10 catheter could not be advanced fully due to concern over causing spasm or dissection. The catheter was replaced by a Marathon microcatheter that was navigated through the

${ }^{\star}$ Correspondence to: Hekmat Zarzour, Assistant Professor of Neurological Surgery, Vickie and Jack Farber Institute for Neuroscience Thomas Jefferson University Hospital, USA, Tel: 856-256-7591; Fax: 856-256-7595; E-mail: Hekmat.Zarzour@jefferson.edu

Key words: poyamoya, anterior choroidal artery, aneurysm, embolization

Received: June 12 , 2018; Accepted: June 19, 2018; Published: June 22, 2018 
guide catheter over a Synchro 10 microwire and the anterior choroidal artery was micro-catheterized. The Synchro 10 microwire was removed from the patient and a Mirage 008 Microwire was introduced into the microcatheter in order to navigate distally into the left anterior choroidal artery distal to the anterior choroidal fissure (Figure 5). The microwire and microcatheter were advanced distally towards the aneurysm past the choroidal fissure of the vessel. A negative road map was used to embolize the aneurysm using $0.3 \mathrm{cc}$ of Onyx 34. The microcatheter was removed and repeat angiogram revealed good filing of the AChA (Figure 6), with total occlusion of the aneurysm.

Immediately post-embolization, the patient had a moderate aphasia as well as right sided weakness. She was started on norepinephrine to increase perfusion in the distribution of the left MCA. The patient improved greatly by day 2 , at last follow-up only had a minor hemianesthesia on the right side which was confirmed on a follow-up MRI to correspond to a small area of restricted diffusion in the distal L MCA region.

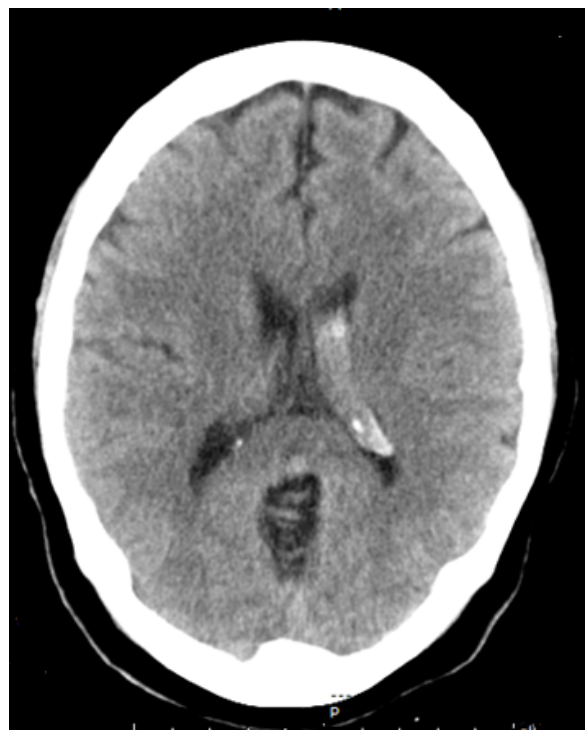

Figure 1. Head CT showing left intra-ventricular bleeding

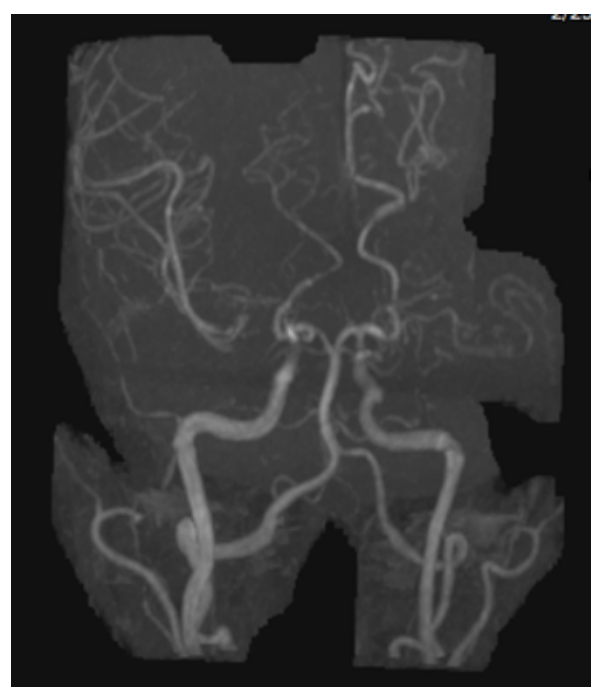

Figure 2. MRA revealed the Moyamoya features. Note the aneurysm in the distal Acha

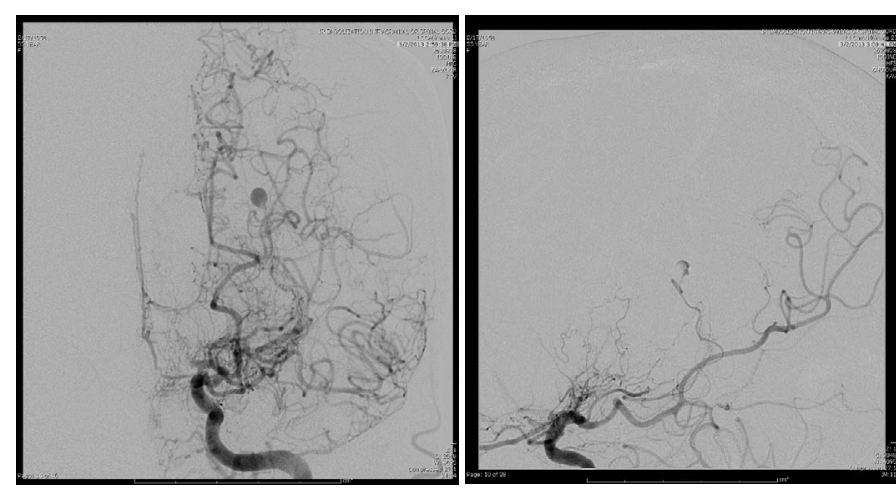

Figure 3. AP and Lateral angiogram showing the aneurysm of distal segment of the anterior choroidal artery (AChA)
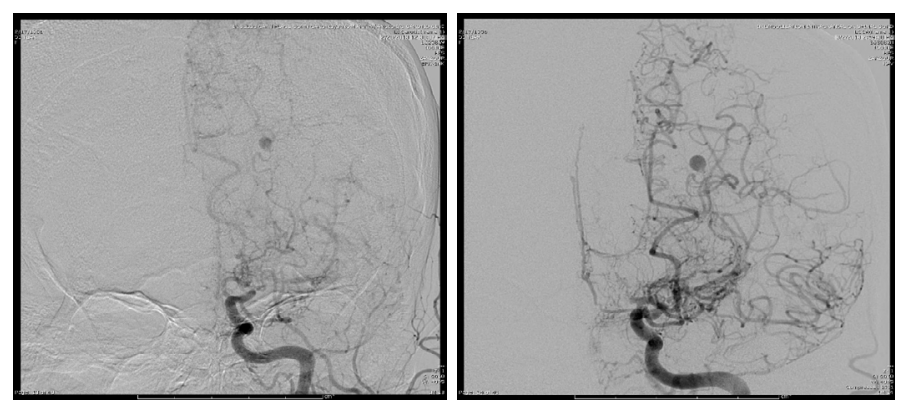

Figure 4. Ap angiogram with 3 days interval showing minimal increased in size
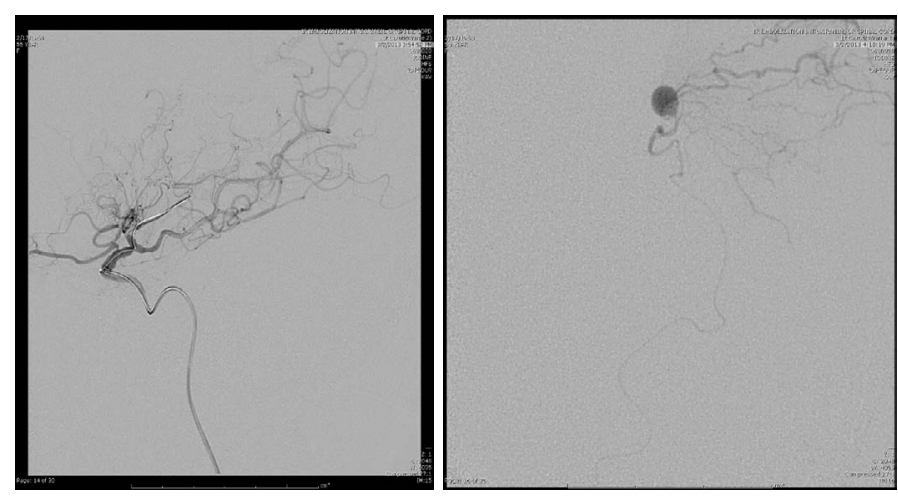

Figure 5. On the left, catheter navigation into the AChA with ICA injection, reveled no aneurysm or AChA filling, which indicate the lumen occlusion of the AChA due to catheterization, followed by micro catheter injection showing the aneurysm with distal perfusion of the cortical arteries
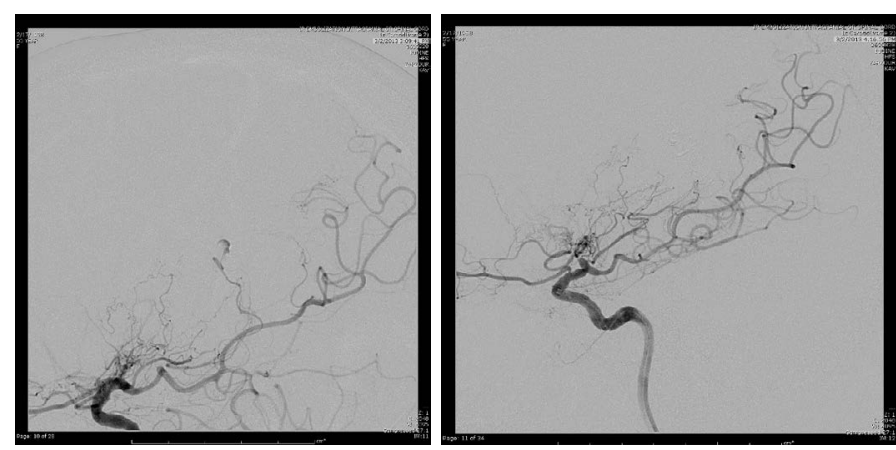

Figure 6. The image on the right showing good filing of the AChA proximal to choroidal fissure with no opacification of the aneurysm 


\section{Discussion}

In this report, we describe one patient with IVH secondary to Moyamoya disease associated with distal AChA aneurysm rupture. Treating a newly diagnosed patient with Moyamoya disease who presented with bleeding secondary to a ruptured aneurysm is challenging, as the aneurysm itself is the result of severe ischemia progression, and the balance between prevent rebleeding and prevent ischemia progression can be tenuous[3-5].

In approaching a patient with Moyamoya and new bleeding, the risk of rebleeding vs conservative or aggressive treatment must be balanced. Xingju et al. [6] reported the long term of re-bleeding after conservative and surgical treatment. They reported 97 patients that were followed for 12 years, 21.7 patients developed rebleeding, and $6.2 \%$ died of such rebleeding over median follow up time of 7.1 years. However in their report, the median interval from initial bleeding to subsequent rebleeding was 9.1 years (0.1-23.2). Patients presenting with hemorrhage has poor outcome, only $45 \%$ of patients had good recovery after first bleeding and $21 \%$ after second, with annual risk of rebleeding of $7 \%$, mortality was $7 \%$ and increased to $29 \%$ after rebleeding [2,7]. Xingju et al. [6] mentioned that the risk in treated group was much lower, $7.4 \%$ comparing to 37.1 in untreated groups. Higher blood pressure can result in a higher risk for re-bleeding; however, it may be necessary to minimize ischemic damage despite causing additional stress on the aneurysm.

The slow progression of Moyamoya results in compensatory perforators collateralizing with distal MCA branches to perfuse the cortical candelabra and distal MCA. This results in the puff of smoke on angiogram that gives the disease its name. These perforators are small arteries though, and cannot endure the high pressure/demand that is required to supplement distal MCA perfusion. This resulting pressure gradient is, in our opinion, the mechanism responsible for aneurysm formation in the context of this disease. Xingju et al. [6] mentioned the rebleeding rate was different when comparing direct bypass vs indirect, which was $0 \%$ vs $28.5 \%$. If direct bypass has a protective effect on rebleeding, this could be explained by aneurysm formation and rupture being due to this abnormal pressure gradient. Peltier et al [4] report the disappearance of a middle cerebral artery aneurysm associated with Moyamoya after successful revascularization. Ni et al. [5] also reported the disappearance of two aneurysms associated with moyamoya in two patients after STA-MCA bypass. In the first patient, the bypass was created three days after bleeding, six months later the follow up angiogram revealed decreased the Moyamoya vessels and the aneurysm was not detected. The other patient had her bypass five days after bleeding, and DSA after ten months confirmed the disappearance of aneurysm and regression of her Moyamoya with good brain perfusion. Dolati et al. [3] reported development of a new $\mathrm{R}$ distal AChA aneurysm 3 years after iatrogenic occlusion of the $\mathrm{R}$
PCA, an angiogram confirmed that the AChA was supplying the R PCA candelabra, through high flow collateralization, further providing evidence of high flow and dynamic stress and aneurysm development.

Occluding the aneurysm without occluding the main vessels is challenging, and probably will not be effective in preventing recurrence; however it should be tried in all cases. Due to a high pressure gradient on such vessels; recurrence is highly likely unless perfusion of the distal branches can also be performed to decrease the impetus for creating such a gradient by treating the ischemic regions. In most cases no strokes were noticed with this approach, as probably collaterals arteries will supply and prevent stroke, however, there is high risk of stroke in the vessels supplied by aneurysm, in other words, if we have good collateral to start with, why the aneurysm will occur.

Treatment of this aneurysm is challenging in the face of fast increasing in size, STA-MCA bypass can be the best option [5] if the aneurysm is stable, and no sign of ischemia. Glue and onyx with endovascular approach is acceptable approach[3,8-10], endoscopic/ surgical ligation/resection was reported as well [11].

\section{References}

1. Rhoton AL Jr (2002) The supratentorial arteries. Neurosurgery 51: S53-120. [Crossref]

2. Ryan, R.W., A. Chowdhary, and G.W. Britz (2012) Hemorrhage and risk of further hemorrhagic strokes following cerebral revascularization in Moyamoya disease: A review of the literature. Surg Neurol Int 3: 72. [Crossref]

3. Dolati P, Sutherland G, Wong J, Hudon M, Goyal MDistal (2012) anterior choroida artery aneurysm following iatrogenic posterior cerebral artery occlusion : a case report and review of literature. Acta Neurochir (Wien) 154: 53-7. [Crossref]

4. Peltier J, Vinchon M, Soto-Ares G, Dhellemmes P (2008) Disappearance of a middle cerebral artery aneurysm associated with Moyamoya syndrome after revascularization in a child: case report. Childs Nerv Syst 24: 1483-1487. [Crossref]

5. Ni W, Xu F, Xu B, Liao Y, Gu Y, et al. (2012) Disappearance of aneurysms associated with moyamoya disease after STA-MCA anastomosis with encephaloduro myosynangiosis. J Clin Neurosci 19: 485-487. [Crossref]

6. Liu X, Zhang D, Shuo W, Zhao Y, Wang R, et al. (2013) Long term outcome after conservative and surgical treatment of haemorrhagic moyamoya disease. $J$ Neurol Neurosurg Psychiatry 84: 258-265. [Crossref]

7. Kobayashi E, Saeki N, Oishi H, Hirai S, Yamaura A (2000) Long-term natural history of hemorrhagic moyamoya disease in 42 patients. J Neurosurg 93: 976-980. [Crossref]

8. Harreld, J.H, A.R. Zomorodi (2011) Embolization of an unruptured distal lenticulostriate aneurysm associated with moyamoya disease. AJNR Am J Neuroradiol 32: E42-3. [Crossref]

9. Yang S, Yu JL, Wang HL, Wang B, Luo Q (2010) Endovascular embolization of distal anterior choroidal artery aneurysms associated with moyamoya disease. A report of two cases and a literature review. Interv Neuroradiol 16: 433-441. [Crossref]

10. Yu JL, Wang HL, Xu K, Li Y, Luo Q (2010) Endovascular treatment of intracrania aneurysms associated with moyamoya disease or moyamoya syndrome. Interv Neuroradiol 16: 240-248. [Crossref]

11. Lévêque M, McLaughlin N, Laroche M, Bojanowski MW (2011) Endoscopic treatment of distal choroidal artery aneurysm. J Neurosurg 114: 116-119. [Crossref]

Copyright: (C2018 Zarzour H. This is an open-access article distributed under the terms of the Creative Commons Attribution License, which permits unrestricted use, distribution, and reproduction in any medium, provided the original author and source are credited. 